\title{
Retinoblastoma cT3b TNM Finding v7
}

National Cancer Institute

\section{Source}

National Cancer Institute. Retinoblastoma cT3b TNM Finding v7. NCI Thesaurus. Code C88763.

One or more complications are present, which may include tumor-associated neovascular or angle closure glaucoma, tumor extension into the anterior segment, hyphema, vitreous hemorrhage, or orbital cellulitis. (from AJCC 7th Ed.) 